\title{
Voltammetric sensor based on bimetallic nanocomposite for determination of favipiravir as an antiviral drug
}

\author{
Mohammad Mehmandoust ${ }^{1,2} \cdot$ Yasamin Khoshnavaz ${ }^{1}$ Mustafa Tuzen ${ }^{3,4} \cdot$ Nevin Erk $^{1,2}$
}

Received: 13 July 2021 / Accepted: 10 November 2021 / Published online: 27 November 2021

( $)$ The Author(s), under exclusive licence to Springer-Verlag GmbH Austria, part of Springer Nature 2021

\begin{abstract}
A novel and sensitive voltammetric nanosensor was developed for the first time for trace level monitoring of favipiravir based on gold/silver core-shell nanoparticles (Au@Ag CSNPs) with conductive polymer poly (3,4-ethylene dioxythiophene) polystyrene sulfonate (PEDOT:PSS) and functionalized multi carbon nanotubes (F-MWCNTs) on a glassy carbon electrode (GCE). The formation of Au@Ag CSNPs/PEDOT:PSS/F-MWCNT composite was confirmed by various analytical techniques, including X-ray diffraction (XRD), ultraviolet-visible spectroscopy (UV-Vis), transmission electron microscopy (TEM), energy-dispersive X-ray spectroscopy (EDX), and fieldemission scanning electron microscopy (SEM). Under the optimized conditions and at a typical working potential of $+1.23 \mathrm{~V}$ (vs. $\mathrm{Ag} / \mathrm{AgCl}$ ), the Au@ Ag CSNPs/PEDOT:PSS/F-MWCNT/GCE revealed linear quantitative ranges from 0.005 to 0.009 and 0.009 to $1.95 \mu \mathrm{M}$ with a limit of detection $0.46 \mathrm{nM}(\mathrm{S} / \mathrm{N}=3)$ with acceptable relative standard deviations (1.1-4.9\%) for pharmaceutical formulations, urine, and human plasma samples without applying any sample pretreatment (1.12-4.93\%). The interference effect of antiviral drugs, biological compounds, and amino acids was negligible, and the sensing system demonstrated outstanding reproducibility, repeatability, stability, and reusability. The findings revealed that this assay strategy has promising applications in diagnosing FAV in clinical samples, which could be attributed to the large surface area on active sites and high conductivity of bimetallic nanocomposite.
\end{abstract}

Keywords COVID-19 · Favipiravir · Voltammetry $\cdot$ Carbon nanotube $\cdot$ Conductive polymer $\cdot$ Biological sample analysis

\section{Introduction}

Mohammad Mehmandoust

mehmandoust@ankara.edu.tr

Nevin Erk

erk@pharmacy.ankara.edu.tr

1 Department of Analytical Chemistry, Faculty of Pharmacy, Ankara University, 06560 Ankara, Turkey

2 Biomaterials, Energy, Photocatalysis, Enzyme Technology, Nano \& Advanced Materials, Additive Manufacturing, Environmental Applications, and Sustainability Research \& Development Group (BIOENAMS R\&D Group), Sakarya University, 54187 Sakarya, Turkey

3 Department of Chemistry, Faculty of Science \& Arts, Tokat Gaziosmanpaşa University, Tr-60250 Tokat, Turkey

4 Research Institute, Center for Environment and Water, King Fahd University of Petroleum and Materials, Dhahran 31261, Saudi Arabia
The pathogen responsible for coronavirus disease 2019 (COVID-19), severe acute respiratory syndrome coronavirus 2 (SARS-CoV-2), has caused unprecedented global morbidity and mortality [1-3]. Most COVID-19 patients have mild to moderate symptoms, but about $15 \%$ experience severe pneumonia, and about $5 \%$ have severe respiratory distress syndrome (ARDS), septic shock, and multiple organ failure [4-6], which causes an enduring threat to the respiratory system. COVID-19 conduced such devastating destruction that the World Health Organization (WHO) explained it as a pandemic and announced it globally in 2019 [7], and anyone can become infected with the virus and spread it in the community. In such cases, one of the primary treatment methods is to use antiviral drugs such as favipiravir, which is approved by the food and drug administration (FDA). Favipiravir (Fig. 1) [8] is widely used as an anti-influenza drug [9] and is effective against several viruses. Recently, there have been 
Fig. 1 Chemical structure of favipiravir

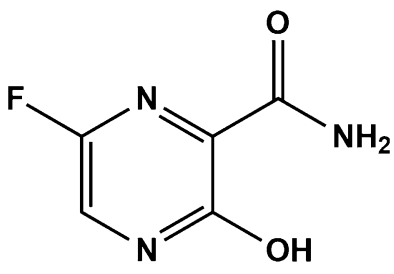

widespread reports of favipiravir to show activity against SARS-CoV-2 [10-13], and in patients with acute conditions, this drug is appropriately used globally. FAV prevents viral replication by selectively inhibiting the RNA polymerase of RNA viruses. It also shows antiviral activity against alpha-, filo-, bunya-, arena-, flavi-, and noroviruses [14, 15]. In addition, there may be a relationship between FAV concentration and clinical response. On the other hand, low plasma FAV concentrations may suggest an inefficient prescription medicine since the levels may be inadequate due to a significant molecular reaction or total cytotoxic response. It also significantly reduces white blood cells. Based on the above results, monitoring the FAV levels in real biological samples has significant importance with a cheap and effective method in clinical diagnostic laboratories.

To date, several analytical methods have been reported for the determination of FAV in biological or pharmaceutical samples, including high-performance liquid chromatography (HPLC) [16], liquid chromatography-tandem mass spectrometry (LC-MS/MS) [17], and spectrofluorimetric method [18], and have been utilized. Among the most widely used instrumental techniques, electrochemical sensors offer an appropriate approach with high catalytic, portability, inexpensive, high chemical stability, and high sensitivity [19-26]. Therefore, electrochemical sensors are widely used to determine trace amounts of drugs and other analytes in pharmaceutical, biological, and environmental samples [27, 28]. One of the most critical steps in an electrochemical sensor is using appropriate materials to improve the performance of the electrode because the bare electrode has a wide range of drawbacks. Thanks to the advances in nanotechnology, multifunctional nanocomposites have been widely employed in wide range of areas [29-34]. Conductive polymers are the type of material with attractive properties used for electrochemical sensors [35]. PEDOT:PSS is an effective organic-electronic material with unique characteristics (such as suitable oxidation potential and bandgap) that can be utilized in various fields, including electrochemical sensors, electrically conducting coatings, and thermoelectric materials [36] because of its mechanical flexibility, appropriate thermal stability, processability, high transparency, excellent electrical conductivity, low price, excellent environmental stability, accessible process able properties, and optical transparency [37-40]. The carbon nanotubes (CNTs) studied so far have shown many beneficial properties that make them attractive for use in sensors and biosensors; the most notable property of these materials is to promote electron transfer reactions [41]. Multi-walled carbon nanotubes have unique mechanical and electronic properties, such as chemical and thermal stability, thermal, and remarkable conductivity. Bimetallic nanoparticles such as Au@Ag can exhibit core-shell structural morphology [42]. The metal nanoparticles (NPs) can be utilized in the host conductive polymer matrix, such as PEDOT:PSS, through the electrochemical method. Using this method, novel properties of the materials are attained, showing synergistic characteristics in terms of their physical and chemical properties [43].

Among the nanomaterials used as a surface modifier in the structure of electrochemical sensors, silver nanoparticles (AgNPs) have attracted attention due to their low toxicity, bio-compatibility, and sustainable electrocatalytic activity [44, 45]. Au nanoparticles are also specially used for surface modification [46, 47]. Besides, the nano-sized AuNPs can increase electrode conductivity, electron transfer, and analytical sensitivity [48]. Gold and silver nanoparticles are frequently used for shell or core materials because of more activity [49-53]. PEDOT:PSS with a higher energy band gap can stabilize the bimetallic core-shell Au@Ag. The electrons and the holes are enclosed within the area of nanocomposite since both conduction band and valence band are found within the shell's energy gap only. The unique structure of functionalized MWCNT increased the specific surface area. The PEDOT:PSS improved the conductivity and enhanced the active sites for the combination and immobilization of Au@Ag CSNPs, which caused good catalytic activity towards the electrochemical oxidation of favipiravir. Electrochemical methods can investigate chemical reactions between the electrode and substances by reacting the electron of importance with the developed surface that produces proportional signals relative to concentrations in the solution.

In this work, Au@Ag CSNPs/PEDOT:PSS/F-MWCNTs were synthesized by hydrogen bonding with PSS and $\pi-\pi$ interaction with PEDOT (Scheme1) (these bonds improve the tensile strength and electrical conductivity) to fabricate the outstanding electrochemical sensor. Au@Ag CS NPs/PEDOT:PSS/F-MWCNT/GCE confirms that notably developed performance to determine trace amount of FAV in real samples, including, human plasma, urine, and tablets, also has satisfactory simplicity and cost-effectiveness. Its sensing and synthesis procedure are systematically exhibited in Scheme 2. The synergic effect of F-MWCNTs, PEDOT:PSS, and metal oxides have remarkably increased the electrocatalytic activity towards the FAV and consequently allowed us to reach its detection limit up to $0.46 \mathrm{nM}$. Au@ Ag CSNPs/ PEDOT:PSS/F-MWCNT/GCE exhibited a promising analytical performance in the wide linear concentration 
Scheme 1 The chemical structure of Au@Ag CSNPs/ PEDOT:PSS/F-MWCNT and its bonds

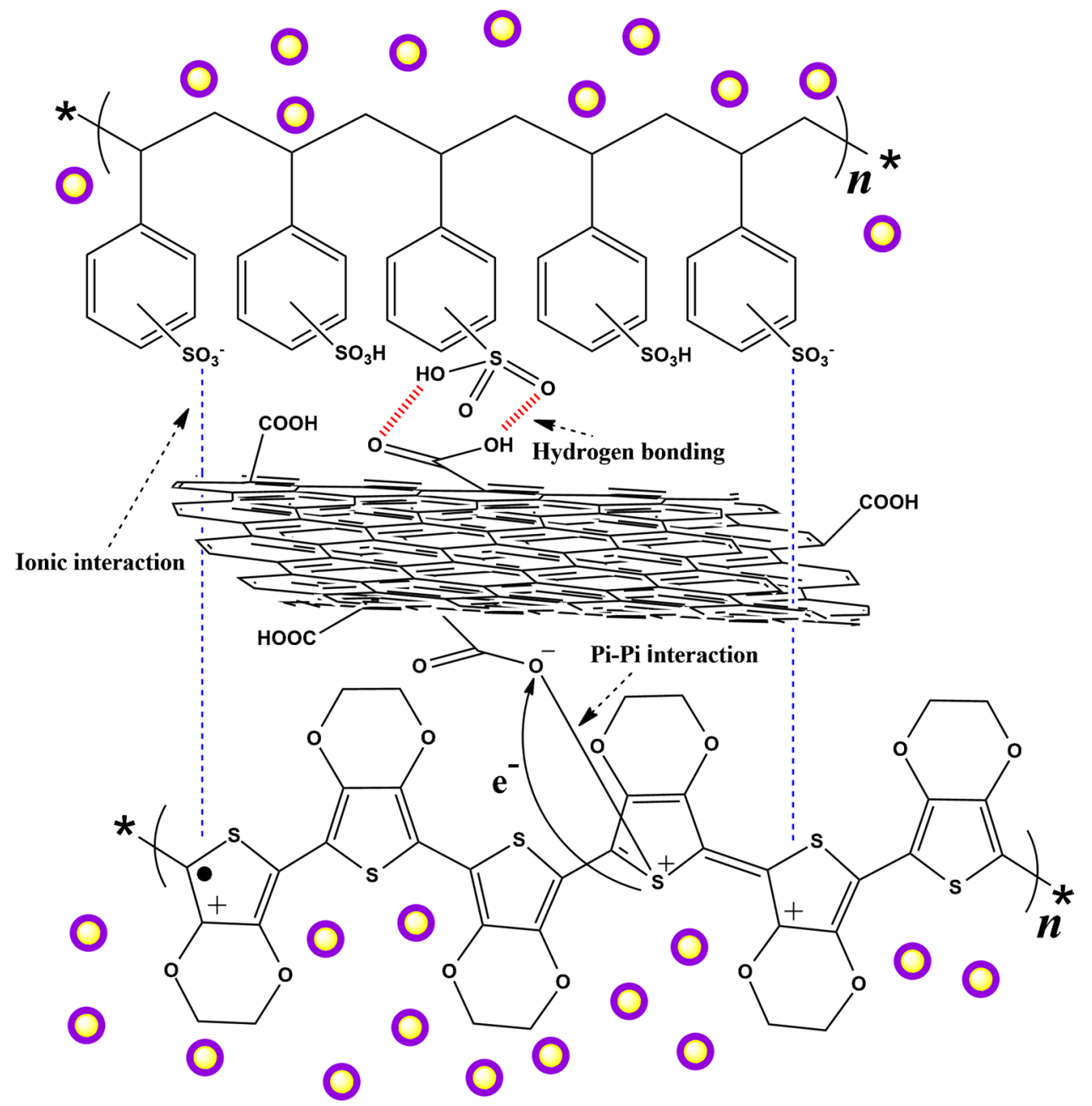

ranges of $0.005-0.009$ and $0.009-2.0 \mu \mathrm{M}$ towards the FAV under optimized experimental conditions. The developed electrochemical sensing platform was successfully utilized to determine FAV in human plasma, urine, and tablet samples with satisfactory recovery.

\section{Experimentals}

\section{Reagents and materials}

Details about reagents and apparatus are described in the Electronic Supporting Material (ESM).

\section{Apparatus}

The electrochemical techniques such as differential pulse voltammetry (DPV), cyclic voltammetry (CV), and chronoamperometry (CA) were conducted using MetrohmAutolab potentiostat/galvanostat system (PGSTAT128N, Netherlands) with a three-electrode system including working electrode: GCE, reference electrode; $\mathrm{Ag} / \mathrm{AgCl}$ (BAS, $3 \mathrm{M} \mathrm{KCl}$ ) and counter electrode; platinum wire. Electrochemical impedance spectroscopy (EIS) was performed under the frequency, $0.1 \mathrm{~Hz}$ to $100 \mathrm{kHz}$ by an IVIUM Compactstat (Eindhoven, Netherlands) device. UV-Vis was recorded using a double beam spectrophotometer (Shimadzu-Japan) model UV-1800 and quartz cells (Hellma-Germany). SEM and EDX were observed micrographs of the materials by ZEISS Gemini SEM 560 at $3.00 \mathrm{kV}$. The X-ray diffraction (XRD) pattern was recorded by Rigaku smart laboratory diffractometer (operated at $40 \mathrm{kV}$ and $20 \mathrm{~mA}$ ). TEM images were performed using an FEI Tecnai G2 Spirit microscope (Oregon, USA) at $120 \mathrm{kV}$.

\section{Preparation of functionalized MWCNTs and gold colloids}

F-MWCNTs and Au colloids were synthesized according to the previously reported procedure $[54,55]$. ESM details the synthesis process of F-MWCNTs and Au colloids. 
Scheme 2 The synthesis procedure and sensing of Au@ Ag CSNPs/PEDOT:PSS/FMWCNT/GCE to determine favipiravir
Step1.

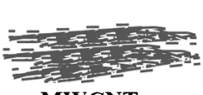
1) $\mathrm{H}_{2} \mathrm{SO}_{4} / \mathrm{HNO}_{3}(3: 1)$

2) $8 \mathrm{~h}$, sonicate 3) $20 \mathrm{~h}, 150^{\circ} \mathrm{C}$,reflux

Step2.
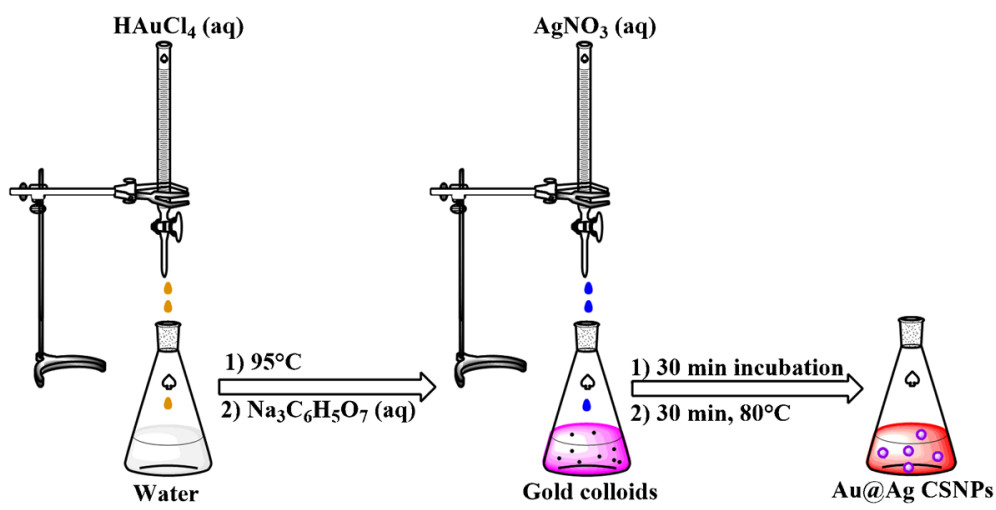

Step3.

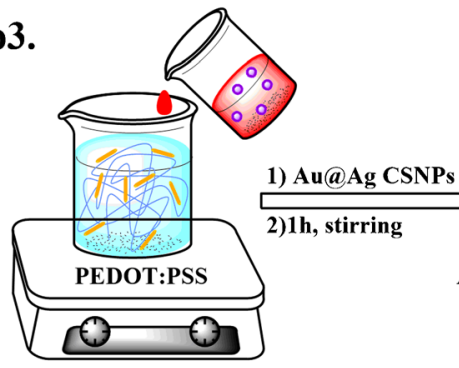

Step4.

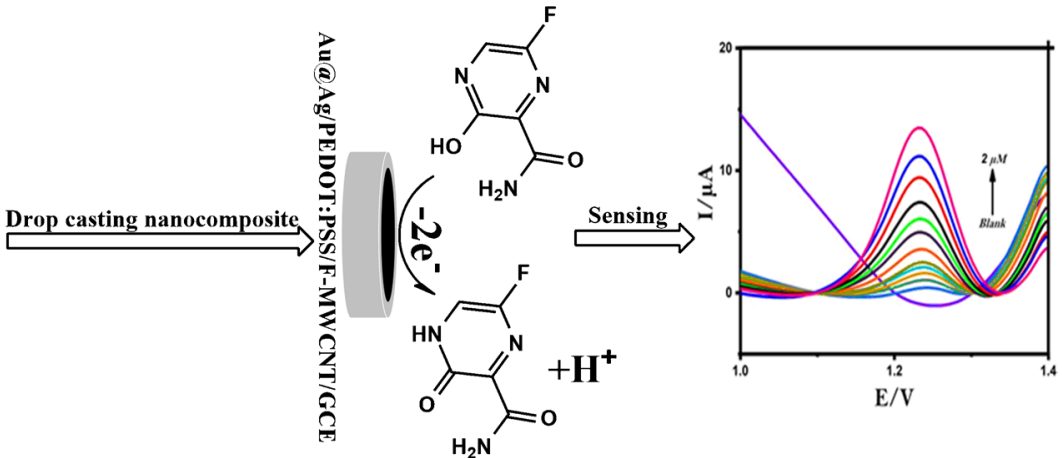

\section{Synthesis and mechanism of the Au@Ag CSNPs}

In $10 \mathrm{ml}$ of the prepared gold colloidal solution, a certain amount of $\mathrm{AgNO}_{3}(10.0 \mathrm{mM})$ was added dropwise. After $30 \mathrm{~min}$ of incubation, the solution was heated to $80{ }^{\circ} \mathrm{C}$ in a water bath. After about $45 \mathrm{~min}$, the pink color of the solution changed to reddish yellow, showing the formation of a silver shell on the gold particles [56]. The synthesized Au@ Ag core-shell nanoparticles were frequently washed with DI water to detach unreacted chemicals through centrifugation and were redispersed in $5 \mathrm{ml}$ of deionized water. The mechanism of core-shell structured Au@Ag CSNPs formation is shown in Scheme 3. $\mathrm{Au}^{0}$ atom sacrificed its electrons to reduce $\mathrm{Ag}^{+}$ions, produce one $\mathrm{Ag}^{0}$ atom, and turn into $\mathrm{Au}^{3+}$

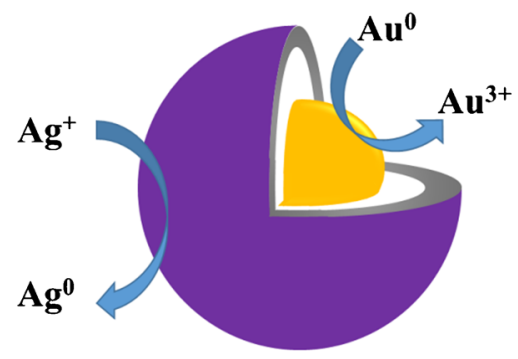

$6 \mathrm{HAuCl}_{4}+\mathrm{Na}_{3} \mathrm{C}_{6} \mathrm{H}_{5} \mathrm{O}_{7}+5 \mathrm{H}_{2} \mathrm{O} \longrightarrow 6 \mathrm{Au}+6 \mathrm{CO}_{2}+21 \mathrm{HCl}+\mathrm{NaCl}$ $\mathrm{Au}^{0}+3 \mathrm{AgNO}_{3} \longrightarrow 3 \mathrm{Ag}^{0}+\mathrm{Au}^{3+}+3 \mathrm{NO}_{3}$ 
ions. This mechanism is repeated as long as $\mathrm{Au}^{3+}$ ions are permeating and electrons are exchanged, resulting in forming a uniform Ag coating on the surface of Au NPs.

\section{Preparation of the Au@Ag CSNPs/PEDOT:PSS/ F-MWCNTs}

The Au@Ag/PEDOT:PSS aqueous dispersion was synthesized by dispersing Au@Ag CSNPs and PEDOT:PSS in $5.0 \mathrm{ml}$ of deionized water under vigorous stirring for $1 \mathrm{~h}$. Finally, 15\% F-MWCNTs (optimized) were dispersed in the 85\% Au@Ag CSNPs/PEDOT:PSS solution by sonication for $10 \mathrm{~h}$ to obtain a homogeneous solution at room temperature [57].

\section{Preparation of Au@Ag CSNPs/PEDOT:PSS/ F-MWCNTs/GCE electrode}

For electroanalytical applications, a GCE was used as working electrodes. For the first $5 \mathrm{~min}$, the electrode surfaces were polished with $0.3 \mathrm{~mm}$ alumina slurries. The alumina residues were then washed in a mixture of ethanol and water $\left(1: 1\right.$, v/v) solution and were dried at $25^{\circ} \mathrm{C}$ for $1 \mathrm{~h}$ under argon gas. Then, 0.75 mg of Au@Ag CSNPs/PEDOT:PSS/ MWCNTs was dispersed in $1.0 \mathrm{ml}$ DI water and sonicating for $30 \mathrm{~min}$. It was then modified by dropping $7.0 \mu \mathrm{L}$ of $\mathrm{Au} @$ Ag CSNPs/PEDOT:PSS/F-MWCNTs onto the clean electrode surface and allowing the modified electrode to dry at room temperature $\left(25^{\circ} \mathrm{C}\right)$. The electrode surface was washed with deionized water to remove the unattached nanocomposite from the electrode surface. All glassware was cleaned by immersing it in $1.0 \mathrm{~mol} \mathrm{~L}^{-1}$ hydrochloric acid and $1.0 \mathrm{~mol}$ $\mathrm{L}^{-1}$ nitric acid for $15 \mathrm{~min}$ and then rinsing it with deionized water. After that, the glassware was dried in a $60{ }^{\circ} \mathrm{C}$ oven [58].

\section{Preparation real samples}

Five Favimol@ tablets (labeled $200 \mathrm{mg}$ per tablet) were completely ground and homogenized. Then, $200 \mathrm{mg}$ of the powders was accurately weighed and diluted with $100 \mathrm{ml}$ of water. Then, the sample solution was filtered via a PTFE syringe filter $(0.45 \mu \mathrm{M})$ and diluted with a buffer to obtain a certain concentration of FAV. The human blood plasma samples were spiked with a certain amount of FAV and $1.0 \mathrm{ml}$ acetonitrile as a precipitating agent. Then, the solution was transferred to centrifugation tubes and made up to the volume with B-R buffer ( $\mathrm{pH} 4.0)$. The precipitated proteins were separated by centrifugation for $15.0 \mathrm{~min}$ at $8000 \mathrm{rpm}$. The urine samples were filtered thoroughly using PTFE syringe filter $(0.45 \mu \mathrm{m})$ membrane filters for the urine sample preparation. A certain amount of FAV solution was added to the urine solution to prepare FAV-spiked urine samples. Finally, the standard addition method was applied to determine FAV in real samples.

\section{Result and discussion}

\section{Choice of materials}

At present, the bimetallic nanoparticles attract high attraction among the researchers due to their excellent electronic conductivity and good electrochemical performances. Recently, the researchers have been using other materials to increase the active sites of metal nanoparticles in a composite on the electrode surface which can boost its activity. Significantly, the sensors based on the conductive polymer sensing materials have shown a promising application in the electrochemical approach. To obtain a highly sensitive and selective sensor, three materials, including Au@ Ag CSNPs, PEDOT:PSS, and F-MWCNTs, were selected to modify
Fig. 2 A UV-Vis absorption spectra of PEDOT:PSS (black line) and Au@Ag CSNPs (red line). B XRD patterns of F-MWCNT and Au@ Ag CSNPs/PEDOT:PSS/FMWCNT
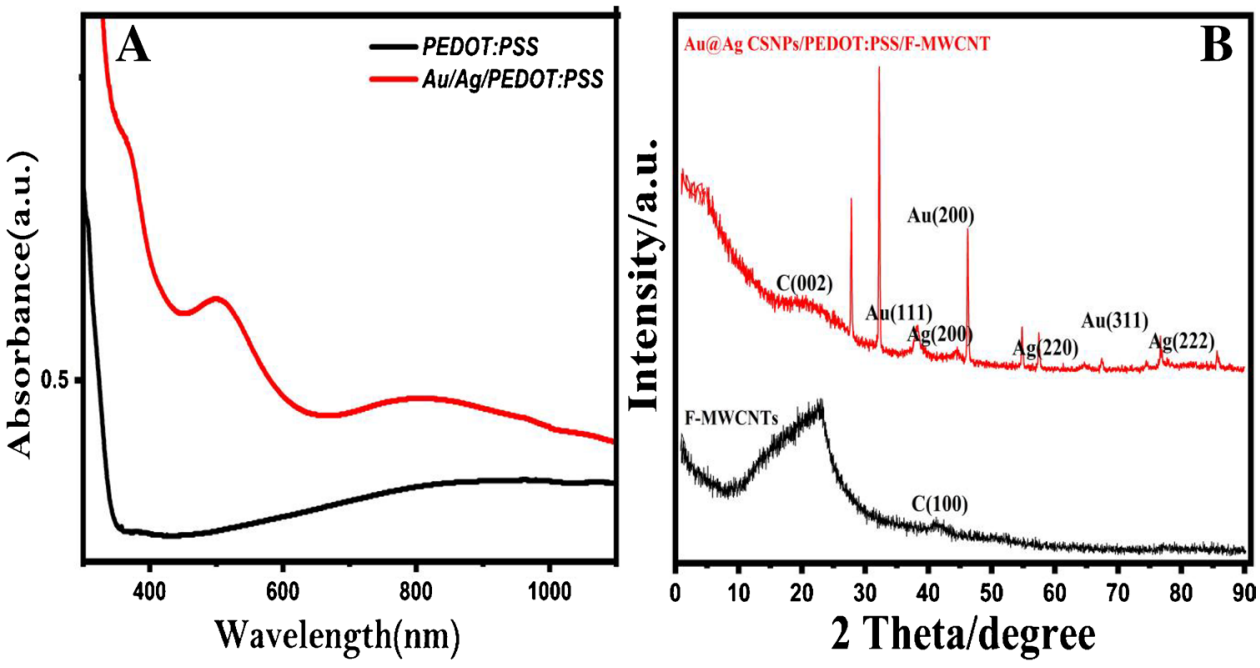
GCE. Au@Ag CSNPs were chosen due to their excellent electrical conductivity. In addition, F-MWCNTs, because of having active groups on their surface, was utilized to improve the sensitivity of the sensor from the enhancement of electron transfer and the enrichment of FAV towards the electrode surface. Therefore, we chose to design a sensitive platform based on the bimetallic composite to determine the FAV by an oxidation mechanism that occurs on the electrode surface.

\section{Characterization of Au@Ag CSNPs/PEDOT:PSS/ F-MWCNTs}

The UV-Visible absorption spectrum of PEDOT:PSS and Au@Ag/PEDOT:PSS is shown in Fig. 2A and was utilized to observe the properties of Au@Ag CSNPs. The PEDOT:PSS showed no adsorption at 300 to 100 wavelengths (black line) [59]. The peak of the Ag@Au CSNPs (red line) is shifted mainly to the near-infrared region $(815 \mathrm{~nm})$ [60]. This significant shift is due to the considerable variation in the collective oscillations of the surface free electrons after Ag-shell formation and the presence of polaron and bipolaron charge carriers, and the oxidation state of the conductive polymer PEDOT [61]. Ag NPs on the surface of Au NPs were demonstrated by the extension of the characteristic absorption peak, which confirms the production of Au@Ag bimetallic nanoparticles.

Figure 2B exhibits the XRD pattern of the Au@Ag CSNPs/PEDOT:PSS/F-MWCNTs and F-MWCNTs. XRD pattern of F-MWCNTs is demonstrated in Fig. 2B. Typical (002) and (100) peaks of F-MWCNT were attained at $2 \theta=26.2^{\circ}$ and $43.4^{\circ}$, respectively [62]. Figure $2 \mathrm{~B}$ shows an XRD image of Au@Ag CSNPs/PEDOT:PSS/F-MWCNTs. Due to similar lattice constants of $\mathrm{Au}$ and $\mathrm{Ag}$ (4.078 and $4.086 \AA$, respectively), it is a little difficult to distinguish them [63]. The pristine PEDOT:PSS has no sharp peak, indicating the many amorphous existences of the PEDOT:PSS [64]. Seven additional diffraction peaks can be readily detected in the XRD image of Au@ Ag CSNPs/PEDOT:PSS/ F-MWCNT nanocomposites at $26.2^{\circ}, 38.2^{\circ}, 44.3^{\circ}, 46.2^{\circ}$, $65.5^{\circ}, 77.2^{\circ}$, and $86.1^{\circ}$, respectively. The crystal planes of $\mathrm{C}$ (002) Au (111), Au (200), Au (200), Ag (220), Au (311), and $\mathrm{Ag}$ (222) are in strong agreement with the seven diffraction peaks. The peak of F-MWCNTs at $43.4^{\circ}$ was not found to
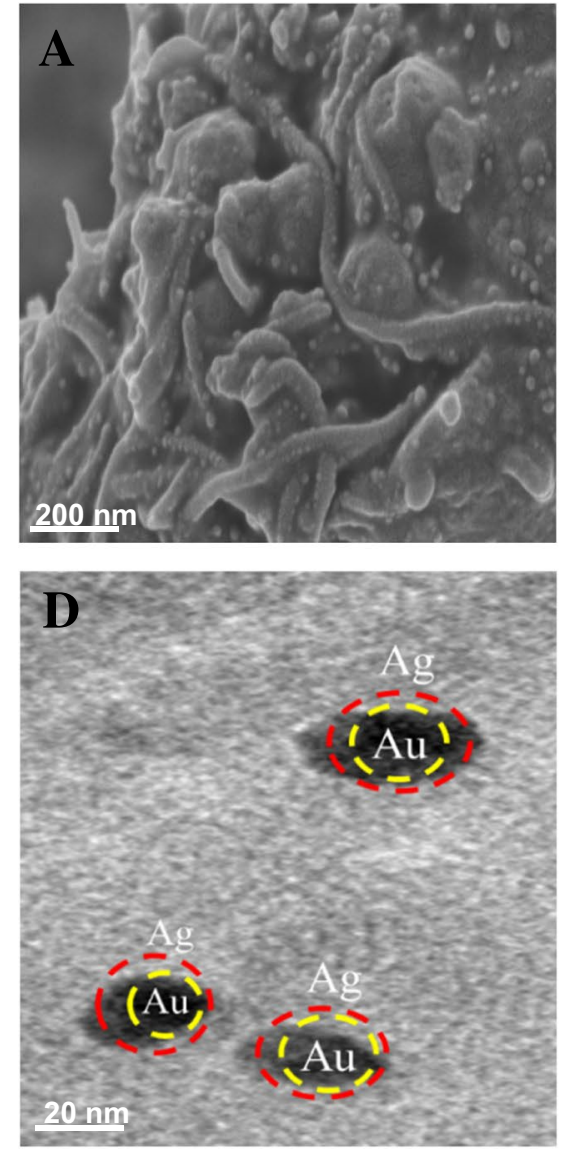
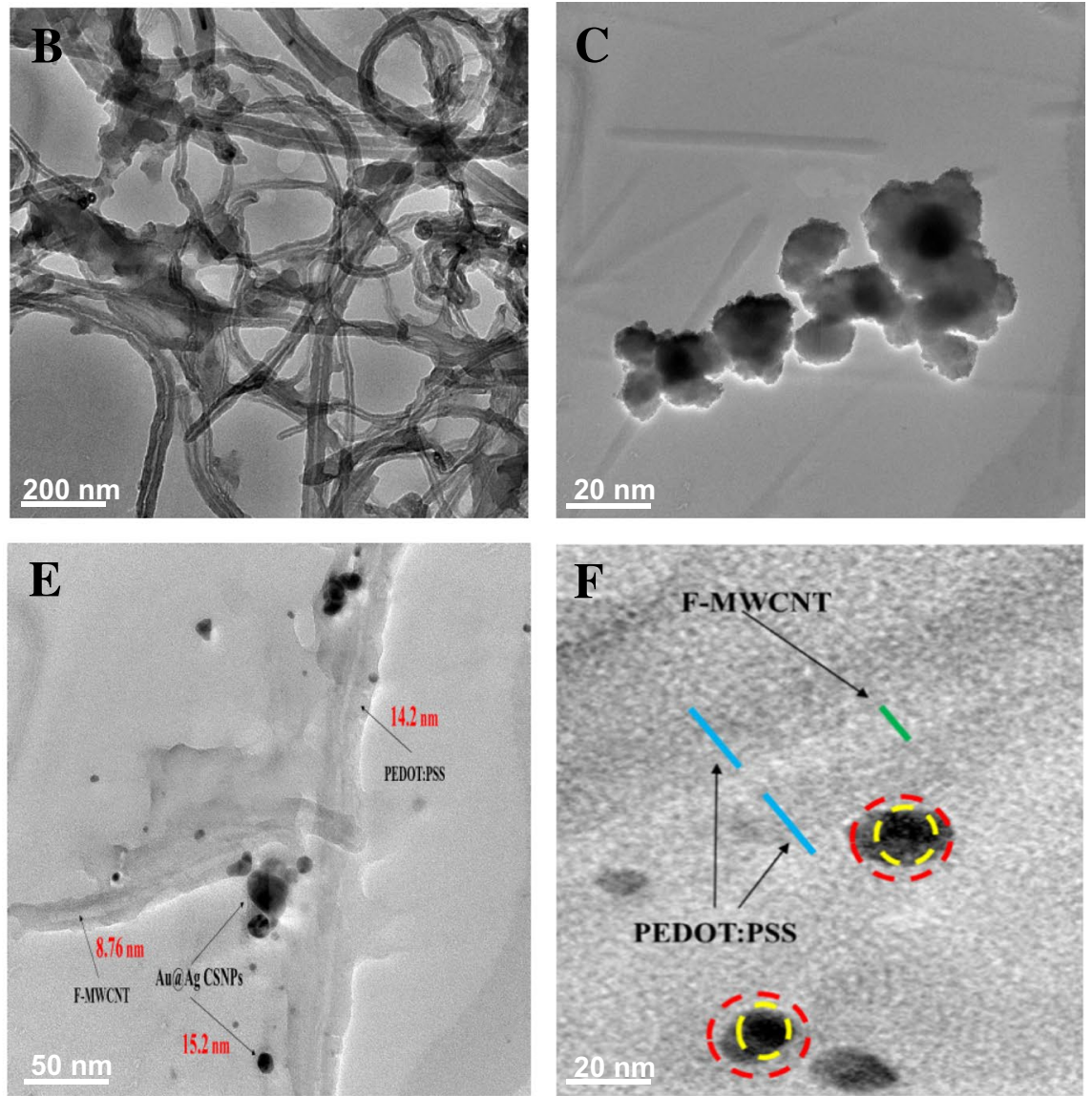

Fig. 3 SEM images of Au@Ag CSNPs/PEDOT:PSS/F-MWCNT (A) and TEM images of F-MWCNT (B), Au@Ag CSNPs (C, D), and Au@Ag CSNPs/PEDOT:PSS/FMWCNT (E, F) 
be probably due to the $\pi-\pi$ interaction between F-MWCNTs and the thiophene rings PEDOT:PSS.

SEM's surface morphologies of the Au@Ag CSNPs/ PEDOT:PSS/F-MWCNTs and F-MWCNTs were observed. As observed in Fig. S1A, the F-MWCNTs exhibited a stable, smooth, and homogeneous structure. The SEM of PEDOT:PSS/MWCNTs was also observed which shows that the MWCNTs have been covered by PEDOT:PSS (Fig. S1B).

SEM image of the Au@Ag CSNPs/PEDOT:PSS/ MWCNTs revealed nanoparticles of core-shell Au@Ag (Fig. 3A).Au@Ag CSNPs were homogeneously distributed on the surface of the PEDOT:PSS and MWCNTs. The effective increase of surface area due to the Au@Ag CSNPs could be effective. The SEM micrograph exhibits that F-MWCNTs are uniformly dispersed throughout the polymer of PEDOT:PSS. Moreover, the EDX analysis of MWCNT (Fig. S1C) and Au@Ag CSNPs/ PEDOT:PSS/F-MWCNTs (Fig. S1D) beads indicated the presence of $\mathrm{C}$ and $\mathrm{O}$ with 24.27 and $64.78 \mathrm{wt} \%$, respectively, for F-MWCNT and Ag, Au, O, and C with 15.24, 4.69, 30.29, and 34.31 wt\%, respectively, for Au@ Ag/ PEDOT:PSS/F-MWCNTs.

As shown in Fig. 3B, TEM of functionalized MWCNT indicated the pure formation of CNT with the size of $14.12 \mathrm{~nm}$ without any amorphous carbon. TEM image of F-MWCNT exhibited no effect on its surface morphology. Therefore, F-MWCNT could be used to synthesize nanocomposite. Figure 3C, D clearly shows $\mathrm{Au}$ and Ag

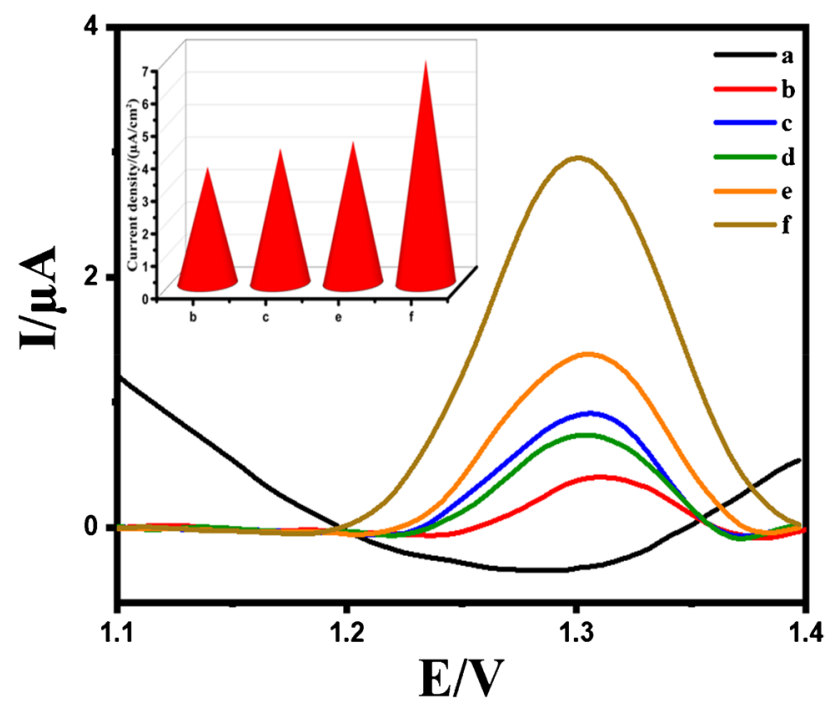

Fig. 4 DPV of Au@Ag CSNPs/PEDOT:PSS/F-MWCNT/GCE in blank (a); bare GCE (b), PEDOT:PSS/GCE (c); Au@Ag/GCE (d); F-MWCNTs/GCE (e); and Au@Ag CSNPs/PEDOT:PSS/FMWCNT/GCE (f) in the presence of $1.0 \mu \mathrm{M} \mathrm{FAV}$ at $\mathrm{pH} 4.0$, insert, current density diagrams obtained from differential pulse voltammograms data core-shell structure for Au@Ag CSNPs. Figure 3E, F exhibits TEM of Au@Ag/PEDOT:PSS/F-MWCNT could be seen that MWCNT was coated with a polymer of PEDOT:PSS. It also revealed that Au NPs (core) coated with Ag NPs (shell). The PEDOT:PSS size was about $14.2 \mathrm{~nm}$, and the size of Au@Ag CSNPs was estimated to be $15.2 \mathrm{~nm}$.

\section{Electrochemical behavior of Au@Ag CSNPs/ PEDOT:PSS/F-MWCNTs/GCE}

The electrocatalytic oxidation of FAV was analyzed by differential pulse voltammetry (DPV). Figure 4 shows the well-defined oxidation peaks of the bare, and modified electrode in 0.1 M B-R buffer ( $\mathrm{pH} 4.0$ ) in the presence of $1.0 \mu \mathrm{M}$ FAV. The oxidation peak currents of blank solution (a), bare electrode (b), PEDOT:PSS/GCE (c), Au@ Ag/GCE (d), F-MWCNTs/GCE (e), and Au@Ag CSNPs/ PEDOT:PSS/F-MWCNTs/GCE (f) are 0.0, 0.21, 0.79, $0.70,1.38$, and $3.12 \mu \mathrm{A}$, respectively. Furthermore, the corresponding voltametric curves represented by DPV demonstrated that the modification of GCE surfaces by bimetallic-based composites remarkably enhanced the sensitivity towards the FAV with a good electrocatalytic activity (i.e., from 1317 to $1295 \mathrm{mV}$ ) compared to that of bare GCE due to their large surface areas. These observations clearly suggest a synergistic effect of the combination of the Au@Ag CSNPs, PEDOT:PSS, and F-MWCNT hybrids in the electrode composition. These results confirmed that Au@Ag CSNPs/PEDOT:PSS/F-MWCNTs/ GCE has the highest electrocatalytic activity towards the oxidation of FAV for the further electrochemical analysis of FAV. The excellent electrocatalytic behavior of Au@ Ag CSNPs/PEDOT:PSS/F-MWCNTs might be due to the rapid electrode transfer rate on the modified electrode surface. Furthermore, the Randles-Sevcik equation (Eq. 1) was used to calculate the electrode active surface area of the sensing electrode $[65]$ using $5.0 \mathrm{mM}\left[\mathrm{K}_{3}(\mathrm{CN})\right]_{6}{ }^{3-/-4}$ and $0.1 \mathrm{M} \mathrm{KCl}$. Based on the slope of the plot (Fig. S2) $\mathrm{I}_{\mathrm{pa}}$ vs. $v \frac{1}{2}$, the active electrode area was obtained using the following equation:

$I_{p a}=\left(2.69 \times 10^{5}\right) n^{\frac{3}{2}} A D^{\frac{1}{2}} v^{\frac{1}{2}} C_{0}$

$A$ represents the electrode area in $\mathrm{cm}^{2}, D_{0}$ shows the diffusion coefficient $\left(\mathrm{cm}^{2} / \mathrm{s}\right), n$ is numbers of electrode $(n=1)$, $v$ exhibits potential scan $\mathrm{rate}(\mathrm{V} / \mathrm{s})$, and $C_{0}$ is concentration $\left(\mathrm{mol} / \mathrm{cm}^{3}\right)$. Therefore, the electrode active area of Au@ Ag CSNPs/PEDOT:PSS/F-MWCNTs/GCE, F-MWCNTs/ GCE, PEDOT:PSS/GCE, and bare electrode were found to be $0.45,0.32,0.19$, and $0.054 \mathrm{~cm}^{2}$, respectively, indicating that Au@Ag CSNPs/PEDOT:PSS/F-MWCNTs/GCE 

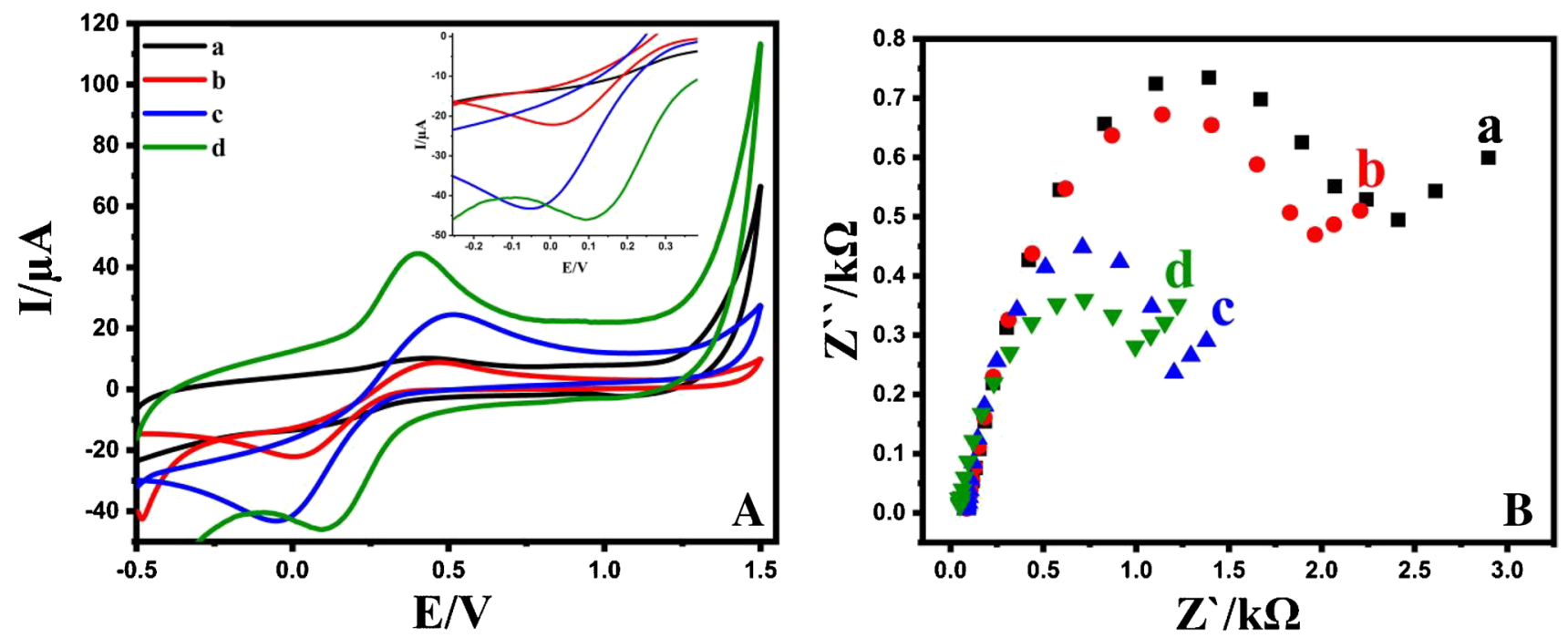

Fig. 5 (A) CVs and (B) Nyquist plot of EIS for bare electrode (a), PEDOT:PSS/GCE (b), F-MWCNT/GCE (c), and Au@Ag CSNPs/ PEDOT:PSS/F-MWCNT/GCE (d) in the presence of $5.0 \mathrm{mM}\left[\mathrm{K}_{3}(\mathrm{CN})\right]_{6}{ }^{3-l-4}$ containing $0.1 \mathrm{M} \mathrm{KCl}$

has promising surface area towards the oxidation of FAV. Therefore, substantiating the incorporation of Au@Ag CSNP and F-MWCNTs with PEDOT:PSS increased the effective surface area of the working electrode by about eightfold.

The electrochemical performance of the developed electrochemical sensor was also studied by determining the electron transfer rate using $\left[\mathrm{K}_{3}(\mathrm{CN})\right]_{6}{ }^{3-/-4}$ solution as a redox probe molecule in $0.1 \mathrm{M} \mathrm{KCl}$. For this purpose, the peak-to-peak separation ( $\Delta \mathrm{Ep}$ ) value between anodic and cathodic signals, which is an essential indicator for electron transfer rate, was determined at the surface of bare GCE, PEDOT:PSS/GCE, and F-MWCNT/ GCE Au@Ag CSNPs/PEDOT:PSS/F-MWCNTs/GCE with a scan rate of $50.0 \mathrm{mV} \mathrm{s}^{-1}$. As seen in Fig. $5 \mathrm{~A}, \Delta \mathrm{Ep}$ value of Au@Ag CSNPs/PEDOT:PSS/F-MWCNTs/ GCE $(\triangle \mathrm{Ep}=280 \mathrm{mV})$ was found to be lower than that of the bare GCE $(\Delta \mathrm{Ep}=480 \mathrm{mV})$, exhibiting the presence of faster electron transfer. In addition, an improvement obtained at both anodic and cathodic signals has clearly revealed the strong electrocatalytic activity of $\mathrm{Au} @ \mathrm{Ag}$ CSNPs/PEDOT:PSS/F-MWCNTs/GCE. Moreover, EIS was utilized to analyze the electrochemical properties of the transformed polymeric matrix at each phase of the modification process to identify a polymeric nanocomposite with higher conductivity [66]. The Randles equivalent circuit (Rct) for bare electrode, PEDOT:PSS/GCE, F-MWCNT/GCE, and Au@Ag CSNPs/PEDOT:PSS/ MWCNTs/GCE were observed to be $2554.1 \Omega, 2310.4 \Omega$, $1849.8 \Omega$, and $1209.54 \Omega$, respectively (Fig. $5 \mathrm{~B}$ ). These results showed that the presence of bimetallic nanoparticles could facilitate the electron transfer between the

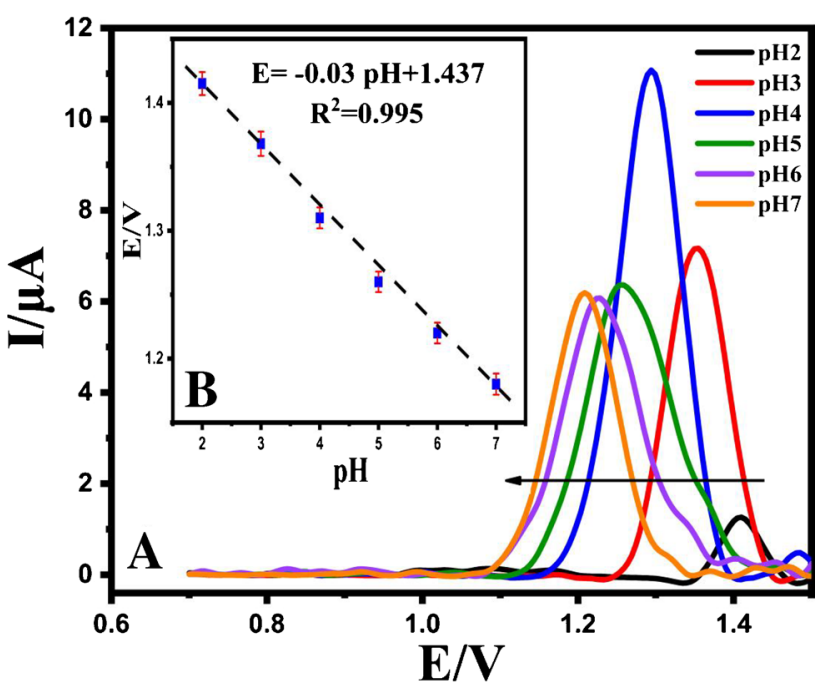

Fig. 6 (A) DPV of $2.0 \mu \mathrm{M}$ favipiravir at the surface of $\mathrm{Au} @ \mathrm{Ag}$ CSNPs/PEDOT:PSS/F-MWCNTs/GCE in the range 2.0-9.0. (B) Epa's plot against $\mathrm{pH}$ for electro-oxidation of $2.0 \mu \mathrm{M}$ favipiravir

analyte and electrode Au@Ag CSNPs/PEDOT:PSS/MWCNTs/GCE, indicating the synergistic effects of MWCNTs, PEDOT:PSS, and Au@Ag CSNPs in advanced nanocomposite using the decrease of the Rct value and increase the electron transfer rate.

\section{Optimization of developed electrode}

The following parameters were optimized (Fig. S3): (A) various supporting electrolytes; (B) concentration of composite; (C) amount of composite. Respective text and figures 
Fig. 7 (A) CVs of $0.1 \mathrm{mM}$ favipiravir at various scan rate ( 2.0 to $200.0 \mathrm{mV} / \mathrm{s}$ ), (B) the plot of $I_{p a}$ vs. $v^{1 / 2},(C)$ the plot of the $\log$ I pa vs. $\log \mathrm{v}$ obtained, and (D) the relationship between Epa vs. $\ln v$ at the surface of Au@Ag CSNPs/PEDOT:PSS/ MWCNTs/GCE
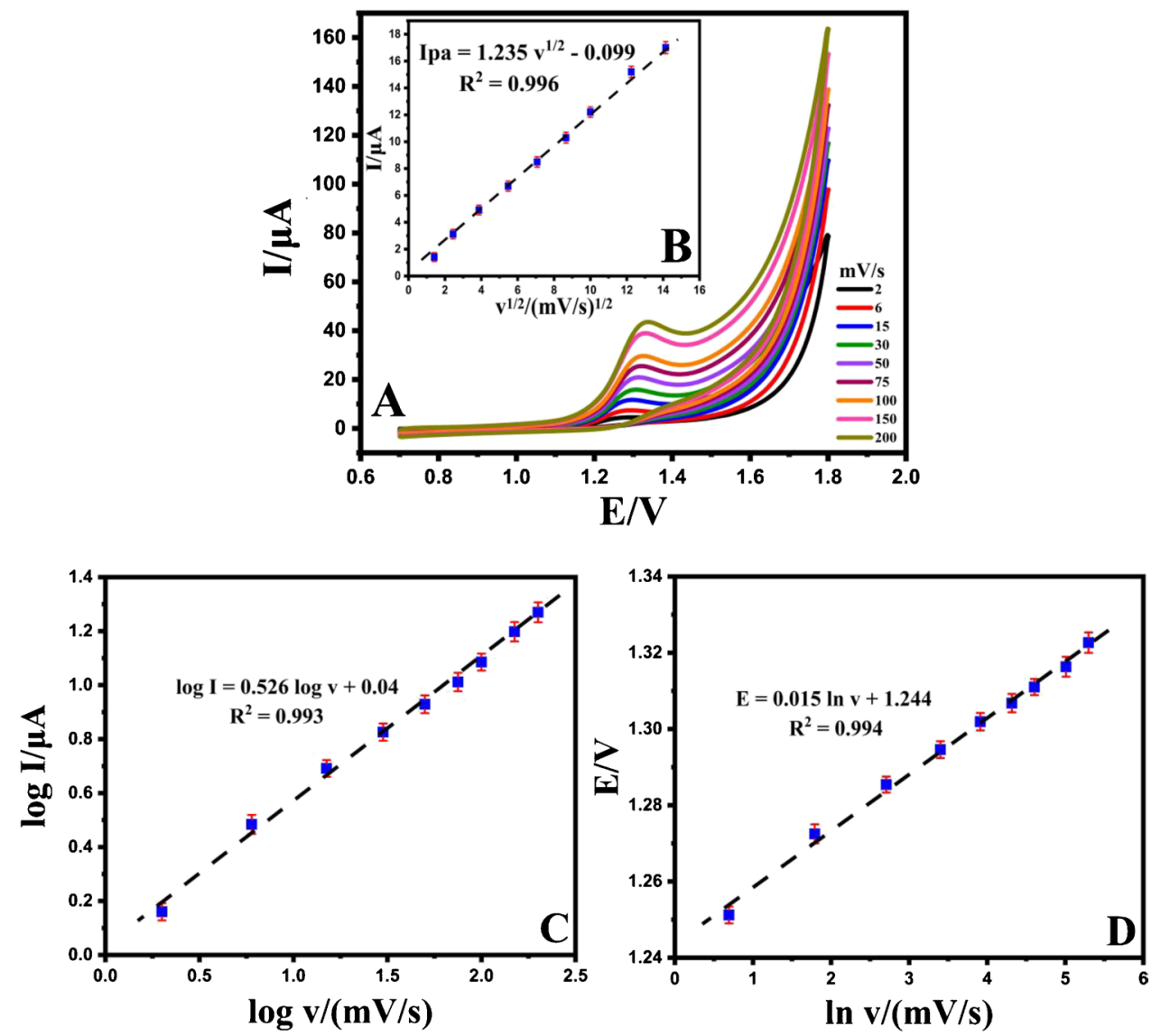

on optimizations are given in the ESM. In short, the following experimental conditions were found to provide the best results: (A) best-supporting electrolyte, B-R buffer; (B) concentration of composite, $0.75 \mathrm{mg} / \mathrm{ml}$; (C) the amount of composite, $7.0 \mu \mathrm{l}$.

Investigation of $\mathrm{pH}$ is a critical method of electrochemical behavior. Therefore, the electrochemical behavior of FAV was applied with DPV in $2.0 \mu \mathrm{M}$ FAV in the presence of $0.1 \mathrm{M}$ B-R buffer with a various $\mathrm{pH}$ range from 2.0 to 9.0 (Fig. 6A). The peak current enhanced with increasing $\mathrm{pH}$ from 2.0 to 4.0 , but the current decreased at $\mathrm{pHs}$ above 4.0 due to FAV being a protic aromatic molecule and has $\mathrm{O}-\mathrm{H}$ group, and the aromatic ring could be a source of protons $\left(\mathrm{H}^{+}\right)$. Whereas $\mathrm{pH}>4$ hydroxyl ions are enhanced, and the adsorption of FAV might be decreased on the electrode surface, the oxidation current dropped at $\mathrm{pH}>4$. Therefore, to achieve high sensitivity, $\mathrm{pH}=4.0$ was selected to detect FAV. The plot of peak potential against $\mathrm{pH}$ as shown in Fig. 6B and the slope value attained $30.0 \mathrm{mV} / \mathrm{pH}$, which is approximately half the theoretical value $(59.0 \mathrm{mV} / \mathrm{pH})$, and the ratio of proton to electron was observed to be $1: 2$.

Dynamic behavior of FAV oxidation was observed by CVs on the developed sensor (Au@Ag CSNPs/PEDOT:PSS/ MWCNTs/GCE) at various scan rates $(2.0-200.0 \mathrm{mV} / \mathrm{s})$. Figure 7A exhibits that the oxidation peak current increases effectively with increasing scan rate. The plot in Fig. 7B exhibits the linear relationship between oxidative peak current and the square root of scan rate, and the equations could be described as $\mathrm{I}_{\mathrm{pa}}(\mu \mathrm{A})=1.235 \mathrm{v}^{1 / 2}-0.099\left(R^{2}=0.996\right)$. It also indicates that the electrocatalytic oxidation of FAV on the developed sensor is a typical diffusion-controlled process [67]. Also, the plot of the logarithm of the oxidation peak current $(\log$ Ipa) vs. the logarithm of the scan rate ( $\log v$ ) was found to be linear with the following regression equation: $\log \operatorname{Ipa}(\mu \mathrm{A})=0.526 \log \mathrm{v}\left(\mathrm{mV} \mathrm{s}^{-1}\right)+0.04$ $\left(R^{2}=0.993\right)$, indicating a diffusion-controlled electrode reaction (Fig. 7C). It is also observed that oxidation peak potential (Epa) of FAV shifted towards the positive direction with the increase of the scan rate, which suggests the expected result for an irreversible electrode reaction [68]. The number of electrons transferred in the oxidation step of FAV can be calculated by Laviron's equation from the slope of Epa vs. natural logarithm of scan rate (ln v) plot (Fig. 7D). The relationship between Epa vs. In $v$ was expressed as follows: $\operatorname{Epa}(\mathrm{V})=0.015 \ln \mathrm{v}+1.244\left(R^{2}=0.994\right)$. The number of electrons in the electro-oxidation of FAV is calculated to be $\sim 2$, demonstrating that the oxidation reaction of FAV at Au@Ag CSNPs/PEDOT:PSS/F-MWCNTs/GCE has two electron transfer process. Therefore, from the results obtained in scan rate and $\mathrm{pH}$ scan, which confirm each other 

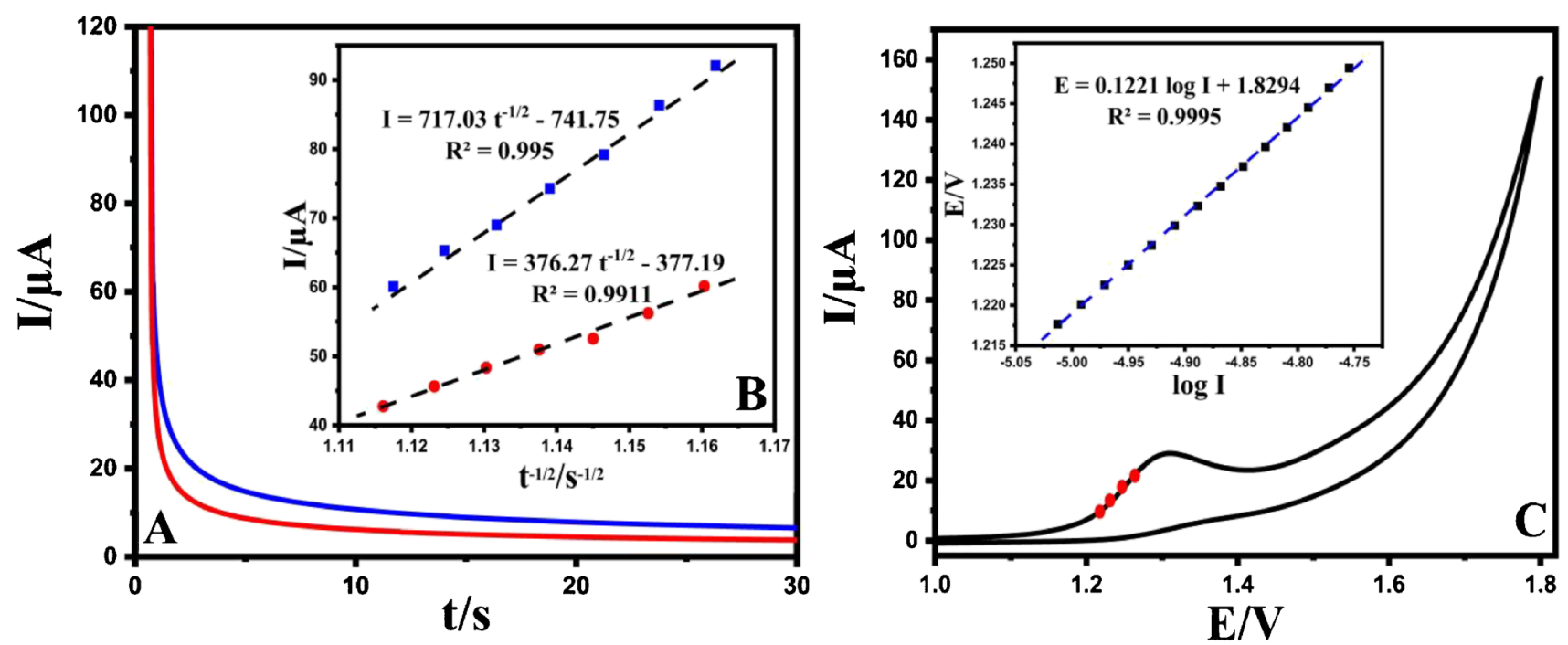

Fig. 8 (A) Chronoamperograms of Au@Ag CSNPs/PEDOT:PSS/FMWCNTs/GCE in the solution containing (a) 500.0 and (b) $600.0 \mu \mathrm{M}$ favipiravir; (B) Cottrell plots obtained from chronoamper-

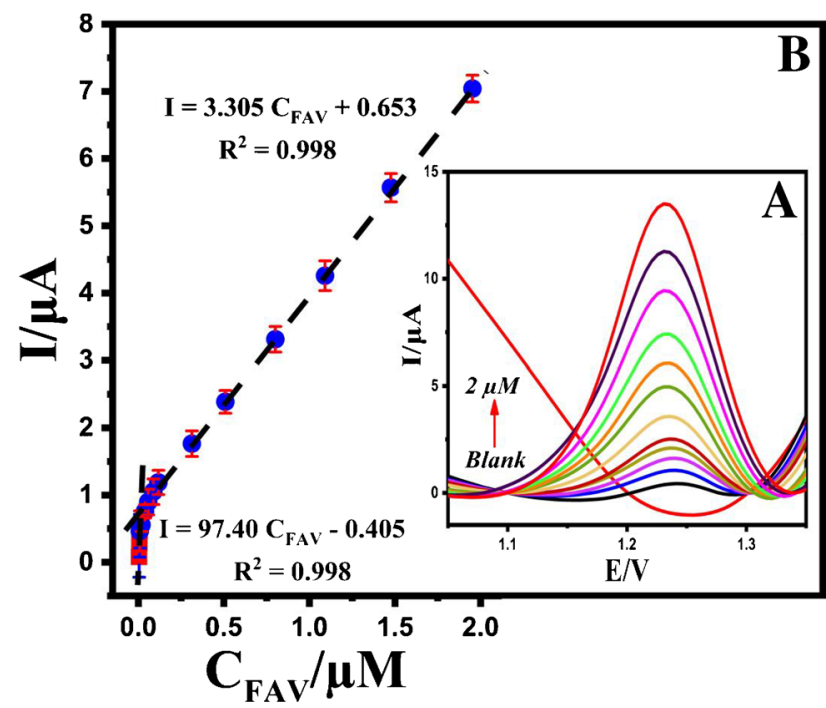

Fig. 9 (A) DPV of favipiravir with various concentrations of FAV ranging from 0.005 to $2.0 \mu \mathrm{M}$ obtained at Au@ Ag CSNPs/ PEDOT:PSS/F-MWCNTs/GCE in pH 4.0 B-R at+1.23 V. (B) Inset: the plot of anodic peak current vs. FAV concentration $(n=3)$ ometry; (C) Tafel plot for Au@Ag CSNPs/PEDOT:PSS/F-MWCNTs/ GCE in B-R buffer ( $\mathrm{pH} 4.0$ ) with a scan rate of $100.0 \mathrm{mV} / \mathrm{s}$ in the presence of $0.1 \mathrm{mM}$ favipiravir

in the number of electrons transferred, reaffirm the mechanism oxidation in Scheme 2, step4.

Moreover, according to the Nernst equation [69], the $\mathrm{m} / \mathrm{n}$ (proton number/electron number) ratio was obtained at 1:2. It is expected that this corresponds to two electrons and one proton involved during the oxidation of favipiravir, as shown in Scheme 2, step 4.

Figure $8 \mathrm{~A}$ exhibits a chronoamperometric investigation of 500.0 and $600.0 \mu \mathrm{M}$ FAV recorded at an applied potential $1.25 \mathrm{~V}$ at a surface of Au@Ag CSNPs/PEDOT:PSS/MWCNTs/GCE. Then, the diffusion coefficient (D) of FAV was calculated to be $8.54 \times 10^{-4}\left(\mathrm{molcm}^{3}\right)$ using the Plot of $\mathrm{I}_{\mathrm{pa}}$ vs.t $\mathrm{t}^{-1 / 2}$ (Fig. 8B) and Cottrell equation (Eq. 2).

$I=n F A C_{b} D^{\frac{1}{2}} \pi^{\frac{-1}{2}} t^{\frac{-1}{2}}$

The bulk concentration $\left(\mathrm{mol} \mathrm{cm}{ }^{-3}\right)$ and diffusion coefficient $\left(\mathrm{cm}^{2} \mathrm{~s}^{-1}\right)$ are represented by $\mathrm{C}_{\mathrm{b}}$ and $\mathrm{D}$, respectively.

Figure 8C exhibits the Tafel plot and its relative voltammograms for electro-oxidation of $0.1 \mathrm{mM} \mathrm{FAV}$ at a scan rate of $100 \mathrm{mV} / \mathrm{s}$. Using the slop of Tafel plot $(2.3 \mathrm{RT} / \mathrm{n}(1-\alpha) \mathrm{F})$,
Table 1 Comparison of studies for FAV detection

\begin{tabular}{lllll}
\hline Method & Modified & Linear range $(\mu \mathrm{M})$ & LOD $(\mu \mathrm{M})$ & Ref \\
\hline SWV & CPT-BDD & $0.064-130$ & 0.018 & {$[70]$} \\
SWV & $\mathrm{MnO}_{2}$-rGO/SPE & $0.01-55$ & 0.009 & {$[71]$} \\
HPLC & - & $63.6-636.5$ & 7.6 & {$[16]$} \\
LC-MS/MS & - & $0.63-127.3$ & 0.15 & {$[72]$} \\
DPV & Au@ AgCSNPs/ & $0.005-2$ & 0.0046 & Our work \\
& PEDOT:PSS/F-MWCNTs/ & & & \\
& GCE & & & \\
\hline
\end{tabular}


the value of electron transfer coefficient $(\alpha)$ was observed $\sim 0.75$, which affirms that the activation free energy curve for an irreversible electro-oxidation mechanism is not symmetrical.

\section{Limit of detection and quantification}

Figure 9A exhibits the DPV responses of the Au@Ag CSNPs/PEDOT:PSS/F-MWCNTs/GCE to FAV in the concentrations ranges from 0.005 to 0.009 and 0.009 to $2.0 \mu \mathrm{M}$. The peak current enhanced significantly with an increase in the concentration of FAV. The linear relationship of the peak current vs. concentrations of FAV indicated $\mathrm{I}_{\mathrm{pa}}=97.403 \mathrm{C}_{\mathrm{FAV}^{-}} 0.4052\left(R^{2}=0.9978\right)$ and $\mathrm{I}_{\mathrm{pa}}=3.3046 \mathrm{C}_{\mathrm{FAV}}+0.653\left(R^{2}=0.9976\right)$, at $\mathrm{Au} @ \mathrm{Ag}$ CSNPs/PEDOT:PSS/F-MWCNTs immobilized GCE (Fig. 9B). Because of kinetic limitations, the sensitivity (slope) of the second linear segment for FAV has decreased. Therefore, the limit of detection (LOD) and quantification (LOQ) were observed to be 0.46 and $1.51 \mathrm{nM}$, respectively. To the best of our knowledge, this work is the first attempt to develop an electrochemical sensor based on Au@Ag CSNPs/PEDOT:PSS/F-MWCNTs/GCE to determine FAV in human plasma, urine, and tablet samples. Au@Ag CSNPs/PEDOT:PSS/FMWCNTs/GCE with enhanced electrocatalytic activity and low detection ability could be used as an alternative sensing platform for the efficient determination of FAV in clinical applications.

In addition, Table 1 shows various methods between Au@Ag CSNPs/PEDOT:PSS/F-MWCNTs/GCE and other analysis methods. First of all, the developed sensor in this study exhibits high selectivity and sensitivity $\left(216.4 \mu \mathrm{A} \cdot \mu \mathrm{M}^{-1} \cdot \mathrm{cm}^{-2}\right)$ to determine FAV compared to other analysis methods. On the other hand,

Table 2 Influence of various interferents on the oxidation current and potential of FAV $(1.0 \mu \mathrm{M})$ at Au@Ag CSNPs/PEDOT:PSS/F-MWCNTs/GCE

\begin{tabular}{lll}
\hline Interferents & $\begin{array}{l}\text { Molar concentration ratio } \\
\text { (FAV, interferents) }\end{array}$ & $\begin{array}{l}\text { Oxidation } \\
\text { current change } \\
(\%)\end{array}$ \\
\hline Tenofovir & $1: 100$ & +3.21 \\
Abacavir & $1: 100$ & -2.74 \\
Ribavirin & $1: 100$ & -3.49 \\
Ritonavir & $1: 100$ & +2.98 \\
Ascorbic acid & $1: 300$ & -2.37 \\
Dopamine & $1: 300$ & +2.27 \\
Glucose & $1: 300$ & -0.89 \\
Uric acid & $1: 300$ & +0.49 \\
L-cysteine & $1: 300$ & -1.18 \\
L-arginine & $1: 300$ & -1.67 \\
\hline
\end{tabular}

when we examine the properties of repeatability, reproducibility, stability, reusability, and selectivity, the developed electrode in this study shows more suitable properties for FAV analysis. Such an improvement in the sensor response to detecting FAV can be attributed to the high affinity of the electrode to FAV and the efficient combination of Au@Ag CSNPs and PEDOT:PSS with F-MWCNTs on the electrode surface, which improves the efficiency of FAV for its electrochemical oxidation to show outstanding the analytical signal.

\section{Study of selectivity, reproducibility, repeatability, selectivity, stability, and reusability}

The selectivity, repeatability, reproducibility, stability, and reusability of developed sensors are significant parameters in electrochemical measurement. For investigation of interferences effect on the voltametric determination of FAV at Au@Ag CSNPs/PEDOT:PSS/F-MWCNTs/GCE, the electrochemical behavior of the developed sensor was performed simultaneously and individually at $1.0 \mu \mathrm{M}$ of FAV in the presence of 100 -fold of antiviral drugs (tenofovir, abacavir, and ribavirin) and 300-fold of biological compounds (ascorbic acid, glucose, dopamine, and uric acid) and amino acids (1-cysteine and 1-arginine) as interfering materials in B-R buffer ( $\mathrm{pH} 4.0$ ) (Fig. S4, Table 2). The generated current responses were not significantly affected by interfering agents (the RSD was below $4.0 \%$, correlated with the tolerance limit defined in the selectivity measurements). These results show that the Au@Ag CSNPs/PEDOT:PSS/F-MWCNTs/GCE has an outstanding selectivity to determine FAV in matrix samples and also could be applied for the determination of FAV with several antiviral drugs such as tenofovir and abacavir, simultaneously, without any destructive effect in potentials and currents. Moreover, in order to evaluate the reproducibility of the Au@Ag CSNPs/ PEDOT:PSS/F-MWCNTs/GCE (Fig. S5A), the same electrochemical procedure was examined to evaluate five different responses manufactured electrodes in $0.1 \mathrm{M} \mathrm{B}-\mathrm{R}$ buffer $\mathrm{pH}$ 4.0. The relative standard deviation was $2.80 \%$, indicating a remarkable reproducibility of the Au@ Ag CSNPs/PEDOT:PSS/F-MWCNTs/GCE. The repeatability and stability of Au@Ag CSNPs/PEDOT:PSS/FMWCNTs/GCE were also investigated. The relative standard deviation for six successive measurements of FAV was determined at $3.42 \%$ (repeatability, Fig. S5B). The electrode was kept in the laboratory at room temperature, and the developed electrode exhibited $96 \%$ of its initial response after 50 days. These results revealed that Au@Ag CSNPs/PEDOT:PSS/F-MWCNTs/GCE also has appropriate stability and repeatability and could be 
Table 3 Determination of FAV in real samples

\begin{tabular}{llllll}
\hline Samples & Added $(\mu \mathrm{M})$ & Found $(\mu \mathrm{M})^{\mathrm{a}}$ & Recovery $(\%)$ & RSD $(\%)$ & HPLC \\
\hline Human plasma & 0.04 & $0.39 \pm 0.01^{\mathrm{b}}$ & 98.6 & 3.74 & - \\
& 0.1 & $0.10 \pm 0.02$ & 102.3 & 4.62 & - \\
& 0.7 & $0.69 \pm 0.03$ & 0.99 & 2.26 & - \\
& 0.9 & $0.88 \pm 0.05$ & 97.7 & 3.94 & - \\
Urine & 1.2 & $1.14 \pm 0.04$ & 95.0 & 2.87 & - \\
& 0.1 & $0.96 \pm 0.02$ & 96.0 & 1.15 & - \\
& 0.4 & $0.39 \pm 0.01$ & 98.5 & 2.49 & - \\
& 0.5 & $0.51 \pm 0.04$ & 102.4 & 1.68 & - \\
& 0.7 & $0.68 \pm 0.04$ & 97.1 & 2.14 & - \\
Tablet & 0.9 & $0.90 \pm 0.04$ & 100.0 & 1.12 & - \\
& 0 & $0.19 \pm 0.05$ & - & - & $0.2 \pm 0.1$ \\
& 0.1 & $0.29 \pm 0.01$ & 100.2 & 3.70 & - \\
F-test & 0.2 & $0.39 \pm 0.02$ & 100 & 1.19 & - \\
\hline -test & 0.5 & $0.68 \pm 0.03$ & 99.7 & 4.93 & - \\
& 0.7 & $0.88 \pm 0.04$ & 98.5 & 3.54 & $0.9 \pm 0.08$ \\
& 0.9 & $1.10 \pm 0.08$ & 101.1 & 3.89 & $1.09 \pm 0.09$ \\
& & & & & 0.06 \\
\end{tabular}

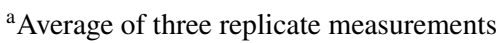

${ }^{\mathrm{b}}$ Mean \pm standard deviation for $n=3$ utilized to determine FAV. Furthermore, the reusability of Au@Ag CSNPs/PEDOT:PSS/F-MWCNTs/GCE was observed.Au@Ag CSNPs/PEDOT:PSS/F-MWCNTs/ GCE is not a disposable sensor. It can be used at least 20 times by washing with $0.1 \mathrm{M} \mathrm{B}-\mathrm{R}$ buffer ( $\mathrm{pH} 4.0$ ). Hence, the reusability of the fabricated sensor surface is required after the twentieth measurement. It was concluded that the developed sensor could be utilized several times and only needs water washing after each analysis.

\section{Investigation of real samples (human plasma, urine, and Tablet $^{\oplus}$ )}

In order to assess the reliability of the proposed electrochemical sensor for the voltametric determination of favipiravir in the real samples was conducted by the standard addition method. As shown in Table 3, the recovery rates and RSD of the Au@Ag CSNPs/ PEDOT:PSS/F-MWCNTs/GCE were observed. The results in Table 3 revealed that the proposed electrochemical sensing platform has sufficient accuracy and precision for FAV determination in real samples. Moreover, the analytical performance of Au@Ag CSNPs/ PEDOT:PSS/F-MWCNTs/GCE was compared to a validated HPLC using Student's $t$-test and $F$-test as a reference method in the Tablet sample. The corresponding values for HPLC demonstrated no significant difference between the developed sensor and the analytical reference method for FAV measurement.

\section{Conclusion}

In the present study, a novel Au@Ag CSNPs/PEDOT:PSS/ F-MWCNT composite was synthesized and characterized using XRD, SEM, TEM, UV-Vis, EIS, and EDX. According to the characterization studies, the obtained nanocomposite exhibited the core-shell structure of AuNPs (core) and AgNPs (shell) well and also coated PEDOT:PSS around CNTs. The novel electrode was tested on the GCE electrode and demonstrated an outstanding sensitivity and selectivity to determine favipiravir in the real samples (Tablet巴, human plasma, and urine). The high sensing property has corresponded to the increased surface area and the synergistic effect between bimetallic core-shell nanoparticles and trapped carbon structures by the conductive polymer. In addition, the modified electrode was observed to be stable, repeatable, reproducible, reusable, and selective. Finally, the developed electrode exhibited high selectivity and sensitivity in real samples compared to other analytical methods. Therefore, it is concluded that this novel electrode could be utilized as a promising method to determine favipiravir in real samples with satisfactory recovery.

Supplementary Information The online version contains supplementary material available at https://doi.org/10.1007/s00604-021-05107-2.

Funding This work was supported by the Scientific Research Projects Commission of Ankara University (Project Number: 21B0237005 and 19L0237004). 


\section{References}

1. Clarke JM, Majeed A, Beaney T, Measuring the impact of covid19 (2021) British Medical Journal Publishing Group. https://doi. org/10.1136/bmj.n1239

2. Alhudhaif A, Polat K, Karaman O (2021) Determination of COVID-19 pneumonia based on generalized convolutional neural network model from chest X-ray images. Expert Syst Appl 180:115141. https://doi.org/10.1016/j.eswa.2021.115141

3. Polat C, Karaman O, Karaman C, Korkmaz G, Balci MC, Kelek SE (2021) COVID-19 diagnosis from chest X-ray images using transfer learning: enhanced performance by debiasing dataloader. J X-Ray Sci Technol 29(1):19-36. https://doi.org/10.3233/ XST-200757

4. Huang C, Wang Y, Li X, Ren L, Zhao J, Hu Y, Zhang L, Fan G, Xu J, Gu X (2020) Clinical features of patients infected with 2019 novel coronavirus in Wuhan, China. Lancet 395(10223):497-506. https://doi.org/10.1016/S0140-6736(20)30183-5

5. Xu Z, Shi L, Wang Y, Zhang J, Huang L, Zhang C, Liu S, Zhao P, Liu H, Zhu L (2020) Pathological findings of COVID-19 associated with acute respiratory distress syndrome. Lancet Respir Med 8(4):420-422. https://doi.org/10.1016/S2213-2600(20)30076-X

6. Cao X (2020) COVID-19: immunopathology and its implications for therapy. Nat Rev Immunol 20(5):269-270. https://doi.org/10. 1038/s41577-020-0308-3

7. Fauci AS, Lane HC, Redfield RR (2020) Covid-19_navigating the uncharted. Mass Med Soc. https://doi.org/10.1056/NEJMe 2002387

8. Furuta Y, Gowen BB, Takahashi K, Shiraki K, Smee DF, Barnard DL (2013) Favipiravir (T-705), a novel viral RNA polymerase inhibitor. Antiviral Res 100(2):446-454. https://doi.org/10.1016/j. antiviral.2013.09.015

9. Hale BG, Albrecht RA, García-Sastre A (2010) Innate immune evasion strategies of influenza viruses. Future Microbiol 5(1):2341. https://doi.org/10.2217/fmb.09.108

10. Kaptein SJ, Jacobs S, Langendries L, Seldeslachts L, Ter Horst S, Liesenborghs L, Hens B, Vergote V, Heylen E, Barthelemy K (2020) Favipiravir at high doses has potent antiviral activity in SARS-CoV-2- infected hamsters, whereas hydroxychloroquine lacks activity. Proc Natl Acad Sci 117(43):26955-26965. https:// doi.org/10.1073/pnas.2014441117

11. Shannon A, Selisko B, Huchting J, Touret F, Piorkowski G, Fattorini V, Ferron F, Decroly E, Meier C, Coutard B (2020) Rapid incorporation of Favipiravir by the fast and permissive viral RNA polymerase complex results in SARS-CoV-2 lethal mutagenesis. Nat Commun 11(1):1-9. https://doi.org/10.1038/ s41467-020-18463-z

12. Boretti A (2020) Favipiravir use for SARS CoV-2 infection. Pharmacol Rep 72(6):1542-1552. https://doi.org/10.1007/ s43440-020-00175-2

13. Kaptein SJ, Jacobs S, Langendries L, Seldeslachts L, ter Horst S, Liesenborghs L, Hens B, Vergote V, Heylen E, Maas E (2020) Antiviral treatment of SARS-CoV-2-infected hamsters reveals a weak effect of favipiravir and a complete lack of effect for hydroxychloroquine. BioRxiv. https://doi.org/10.1101/2020.06. 19.159053

14. De Clercq E (2019) New nucleoside analogues for the treatment of hemorrhagic fever virus infections. Chem Asian J 14(22):3962-3968. https://doi.org/10.1002/asia.201900841

15. Delang L, Abdelnabi R, Neyts J (2018) Favipiravir as a potential countermeasure against neglected and emerging RNA viruses. Antiviral Res 153:85-94. https://doi.org/10.1016/j.antiviral. 2018.03.003
16. Bulduk İ (2021) HPLC-UV method for quantification of favipiravir in pharmaceutical formulations. Acta Chromatogr 33(3):209-215. https://doi.org/10.1556/1326.2020.00828

17. Habler K, Brügel M, Teupser D, Liebchen U, Scharf C, Schönermarck U, Vogeser M, Paal M (2021) Simultaneous quantification of seven repurposed COVID-19 drugs remdesivir (plus metabolite GS-441524), chloroquine, hydroxychloroquine, lopinavir, ritonavir, favipiravir and azithromycin by a two-dimensional isotope dilution LC-MS/MS method in human serum. J Pharm Biomed Anal 196:113935. https://doi.org/10.1016/j.jpba. 2021.113935

18. Megahed SM, Habib AA, Hammad SF, Kamal AH (2021) Experimental design approach for development of spectrofluorimetric method for determination of favipiravir; a potential therapeutic agent against COVID-19 virus: application to spiked human plasma. Spectrochim Acta Part A Mol Biomol Spectrosc 249:119241. https://doi.org/10.1016/j.saa.2020.119241

19. Karaman C, Karaman O, Yola BB, Ulker I, Atar N, Yola ML (2021) A novel electrochemical Aflatoxin B1 immunosensor based on gold nanoparticles decorated porous graphene nanoribbon and $\mathrm{Ag}$ nanocubes incorporated MoS2 nano. New J Chem 45(25):11222-11233. https://doi.org/10.1039/D1NJ02293H

20. Karimi-Maleh H, Karimi F, Fu L, Sanati AL, Alizadeh M, Karaman C, Orooji Y (2022) Cyanazine herbicide monitoring as a hazardous substance by a DNA nanostructure biosensor. J Hazard Mater 423:127058. https://doi.org/10.1016/j.jhazmat.2021.127058

21. Ensafi AA, Dadkhah-Tehrani S, Karimi-Maleh H (2011) A voltammetric sensor for the simultaneous determination of L-cysteine and tryptophan using a p-aminophenol-multiwall carbon nanotube paste electrode. Anal Sci 27(4):409-414. https://doi.org/10.2116/ analsci.27.409

22. Ensafi AA, Karimi-Maleh H, Mallakpour S (2011) N-(3,4Dihydroxyphenethyl)-3,5-dinitrobenzamide-modified multiwall carbon nanotubes paste electrode as a novel sensor for simultaneous determination of penicillamine, uric acid, and tryptophan. Electroanal 23(6):1478-1487. https://doi.org/10.1002/elan.20100 0741

23. Karimi-Maleh H, Orooji Y, Karimi F, Alizadeh M, Baghayeri M, Rouhi J, Tajik S, Beitollahi HD, Agarwal S, Gupta VK, Rajendran S, Ayati A, Fu L, Sanati AL, Tanhaei B, Sen F, Shabani-nooshabadi M, Asrami PN, Al-Othman A (2021) A critical review on the use of potentiometric based biosensors for biomarkers detection. Biosens Bioelectron 184:113252. https://doi.org/10.1016/j.bios. 2021.113252

24. Karimi-Maleh H, Ranjbari S, Tanhaei B, Ayati A, Orooji Y, Alizadeh M, Karimi F, Salmanpour S, Rouhi J, Sillanpaa M, Sen F (2021) Novel 1-butyl-3-methylimidazolium bromide impregnated chitosan hydrogel beads nanostructure as an efficient nanobioadsorbent for cationic dye removal: Kinetic study. Environ Res 195:110809. https://doi.org/10.1016/j.envres.2021.110809

25. Raoof JB, Ojani R, Karimi-Maleh H (2009) Electrocatalytic oxidation of glutathione at carbon paste electrode modified with 2,7bis (ferrocenyl ethyl) fluoren-9-one: application as a voltammetric sensor. J Appl Electrochem 39(8):1169-1175. https://doi.org/10. 1007/s10800-009-9781-x

26. Tajik S, Orooji Y, Karimi F, Ghazanfari Z, Beitollahi H, Shokouhimehr M, Varma RS, Jang HW (2021) High performance of screen-printed graphite electrode modified with Ni-Mo-MOF for voltammetric determination of amaranth. J Food Meas Charact 15(5):4617-4622. https://doi.org/10.1007/s11694-021-01027-0

27. Karaman C, Karaman O, Atar N, Yola ML (2021) Electrochemical immunosensor development based on core-shell high-crystalline graphitic carbon nitride@ carbon dots and Cd0.5Zn0.5S/dTi3C2Tx MXene composite for heart-type fatty acid-binding 
protein detection. Microchim Acta 188(6):182. https://doi.org/ 10.1007/s00604-021-04838-6

28. Karaman C, Karaman O, Yola BB, Ulker I, Atar N, Yola ML (2021) A novel electrochemical aflatoxin B1 immunosensor based on gold nanoparticle-decorated porous graphene nanoribbon and Ag nanocube-incorporated MoS2 nanosheets. New J Chem 45(25):11222-11233. https://doi.org/10.1039/D1NJ02293H

29. Al Sharabati M, Abokwiek R, Al-Othman A, Tawalbeh M, Karaman C, Orooji Y, Karimi F (2021) Biodegradable polymers and their nano-composites for the removal of endocrine-disrupting chemicals (EDCs) from wastewater: a review. Environ Res 202:111694. https://doi.org/10.1016/j.envres.2021.111694

30. Karaman C (2021) Orange peel derived-nitrogen and sulfur codoped carbon dots: a nano-booster for enhancing ORR electrocatalytic performance of 3D graphene networks. Electroanal 33(5):1356-1369. https://doi.org/10.1002/elan.202100018

31. Karaman C, Karaman O, Show PL, Orooji Y, Karimi-Maleh H (2021) Utilization of a double-cross-linked amino-functionalized three-dimensional graphene networks as a monolithic adsorbent for methyl orange removal: equilibrium, kinetics, thermodynamics and artificial neural network modeling. Environ Res: 112156. https://doi.org/10.1016/j.envres.2021.112156

32. Karaman O, Ozdogan H, Uncu VA, Karaman C, Tamar AG (2020) Investigation of the effects of different composite materials on neutron contamination caused by medical LINAC. Kerntechnik 85(5):401-407. https://doi.org/10.3139/124.200022

33. Karimi F, Ayati A, Tanhaei B, Sanati AL, Afshar S, Kardan A, Dabirifar Z, Karaman C (2022) Removal of metal ions using a new magnetic chitosan nano-bio-adsorbent; a powerful approach in water treatment. Environ Res 203:111753. https://doi.org/10. 1016/j.envres.2021.111753

34. Karaman O (2021) Oxygen reduction reaction performance boosting effect of nitrogen/sulfur co-doped graphene supported cobalt phosphide nanoelectrocatalyst: $\mathrm{pH}$-universal electrocatalyst. Ecs J Solid State Sc 10(6):061003. https://doi.org/10.1149/2162-8777/ ac0608

35. Ramanavičius A, Ramanavičienė A, Malinauskas A (2006) Electrochemical sensors based on conducting polymer-polypyrrole. Electrochim Acta 51(27):6025-6037. https://doi.org/10.1016/j. electacta.2005.11.052

36. Wang Z, Xu J, Yao Y, Zhang L, Wen Y, Song H, Zhu D (2014) Facile preparation of highly water-stable and flexible PEDOT: PSS organic/inorganic composite materials and their application in electrochemical sensors. Sens Actuators B Chem 196:357-369. https://doi.org/10.1016/j.snb.2014.02.035

37. Ouyang J, Xu Q, Chu C-W, Yang Y, Li G, Shinar J (2004) On the mechanism of conductivity enhancement in poly (3, 4-ethylenedioxythiophene): poly (styrene sulfonate) film through solvent treatment. Polymer 45(25):8443-8450. https://doi.org/10.1016/j. polymer.2004.10.001

38. Nardes AM, Kemerink M, De Kok M, Vinken E, Maturova K, Janssen R (2008) Conductivity, work function, and environmental stability of PEDOT: PSS thin films treated with sorbitol. Org Electron 9(5):727-734. https://doi.org/10.1016/j.orgel.2008.05. 006

39. Elschner A, Kirchmeyer S, Lovenich W, Merker U, Reuter K (2010) PEDOT: principles and applications of an intrinsically conductive polymer. CRC Press

40. Kim YH, Sachse C, Machala ML, May C, Müller-Meskamp L, Leo K (2011) Highly conductive PEDOT: PSS electrode with optimized solvent and thermal post-treatment for ITO-free organic solar cells. Adv Func Mater 21(6):1076-1081. https:// doi.org/10.1002/adfm.201002290

41. Korkmaz S, Kariper İA, Karaman O, Karaman C (2021) The production of $\mathrm{rGO} / \mathrm{RuO} 2$ aerogel supercapacitor and analysis of its electrochemical performances. Ceram Int. https://doi.org/ 10.1016/j.ceramint.2021.08.366

42. Yao Q, Feng Y, Fung V, Yu Y, Jiang D-e, Yang J, Xie J (2017) Precise control of alloying sites of bimetallic nanoclusters via surface motif exchange reaction. Nat Commun 8(1):1-11. https://doi.org/10.1038/s41467-017-01736-5

43. Hu Z, Patten T, Pelton R, Cranston ED (2015) Synergistic stabilization of emulsions and emulsion gels with water-soluble polymers and cellulose nanocrystals. ACS Sustain Chem Eng 3(5):1023-1031. https://doi.org/10.1021/acssuschemeng.5b001 94

44. Gao Y, Shan D, Cao F, Gong J, Li X, Ma H-y, Su Z-m, Qu L-y (2009) Silver/polyaniline composite nanotubes: one-step synthesis and electrocatalytic activity for neurotransmitter dopamine. J Phys Chem C 113(34):15175-15181. https://doi.org/10.1021/ jp904788d

45. Smausz T, Kecskeméti G, Csizmadia T, Benedek F, Hopp B (2013) Study on the applicability of polytetrafluoroethylene-silver composite thin films as sensor material. Appl Surf Sci 278:117121. https://doi.org/10.1016/j.apsusc.2013.01.051

46. Azzazy HM, Mansour MM, Samir TM, Franco R (2012) Gold nanoparticles in the clinical laboratory: principles of preparation and applications. Clin Chem Lab Med 50(2):193-209. https://doi. org/10.1515/cclm.2011.732

47. Sanghavi BJ, Srivastava AK (2011) Simultaneous voltammetric determination of acetaminophen and tramadol using Dowex $50 \mathrm{w} x 2$ and gold nanoparticles modified glassy carbon paste electrode. Anal Chim Acta 706(2):246-254. https://doi.org/10.1016/j.aca. 2011.08.040

48. Niu X, Yang W, Wang G, Ren J, Guo H, Gao J (2013) A novel electrochemical sensor of bisphenol A based on stacked graphene nanofibers/gold nanoparticles composite modified glassy carbon electrode. Electrochim Acta 98:167-175. https://doi.org/10. 1016/j.electacta.2013.03.064

49. Tsuji M, Miyamae N, Lim S, Kimura K, Zhang X, Hikino S, Nishio M (2006) Crystal structures and growth mechanisms of Au@Ag core- shell nanoparticles prepared by the microwavepolyol method. Cryst Growth Des 6(8):1801-1807. https://doi. org/10.1021/cg060103e

50. Gobin AM, Lee MH, Halas NJ, James WD, Drezek RA, West JL (2007) Near-infrared resonant nanoshells for combined optical imaging and photothermal cancer therapy. Nano Lett 7(7):19291934. https://doi.org/10.1021/nl070610y

51. Yang Y, Shi J, Kawamura G, Nogami M (2008) Preparation of $\mathrm{Au}-\mathrm{Ag}, \mathrm{Ag}-\mathrm{Au}$ core-shell bimetallic nanoparticles for surfaceenhanced Raman scattering. Scripta Mater 58(10):862-865. https://doi.org/10.1016/j.scriptamat.2008.01.017

52. Kalele S, Gosavi S, Urban J, Kulkarni S (2006) Nanoshell particles: synthesis, properties and applications. Curr Sci 91:1038-1052

53. Shankar SS, Rai A, Ahmad A, Sastry M (2004) Rapid synthesis of $\mathrm{Au}, \mathrm{Ag}$, and bimetallic $\mathrm{Au}$ core-Ag shell nanoparticles using Neem (Azadirachta indica) leaf broth. J Colloid Interface Sci 275(2):496-502. https://doi.org/10.1016/j.jcis.2004.03.003

54. Mehmandoust M, Erk N, Alizadeh M, Salmanpour S (2021) Voltammetric carbon nanotubes based sensor for determination of tryptophan in the milk sample. J Food Measur Charact: 1-8. https://doi.org/10.1007/s11694-021-01100-8

55. Dykman LA, Khlebtsov NG (2019) Methods for chemical synthesis of colloidal gold. Russ Chem Rev 88(3):229. https://doi.org/ 10.1070/RCR4843

56. Pande S, Ghosh SK, Praharaj S, Panigrahi S, Basu S, Jana S, Pal A, Tsukuda T, Pal T (2007) Synthesis of normal and inverted gold- silver core- shell architectures in $\beta$-cyclodextrin and their applications in SERS. J Phys Chem C 111(29):10806-10813. https://doi.org/10.1021/jp0702393 
57. Wu T, Pan Y, Bao H, Li L (2011) Preparation and properties of chitosan nanocomposite films reinforced by poly (3, 4-ethylenedioxythiophene)-poly (styrenesulfonate) treated carbon nanotubes. Mater Chem Phys 129(3):932-938. https://doi.org/10. 1016/j.matchemphys.2011.05.030

58. Prakash S, Chakrabarty T, Singh AK, Shahi VK (2013) Polymer thin films embedded with metal nanoparticles for electrochemical biosensors applications. Biosens Bioelectron 41:43-53. https:// doi.org/10.1016/j.bios.2012.09.031

59. Melendez RG, Moreno KJ, Moggio I, Arias E, Ponce A, Llanera I, Moya SE (2010) On the influence of silver nanoparticles size in the electrical conductivity of PEDOT: PSS. in Materials Science Forum. Trans Tech Publ. https://doi.org/10.4028/www.scientific. net/MSF.644.85

60. Dai L, Song L, Huang Y, Zhang L, Lu X, Zhang J, Chen T (2017) Bimetallic Au/Ag core-shell superstructures with tunable surface plasmon resonance in the near-infrared region and high performance surface-enhanced Raman scattering. Langmuir 33(22):5378-5384. https://doi.org/10.1021/acs.langmuir.7b00097

61. Bredas JL, Street GB (1985) Polarons, bipolarons, and solitons in conducting polymers. Acc Chem Res 18(10):309-315

62. Verma D, Chauhan D, Mukherjee MD, Ranjan KR, Yadav AK, Solanki PR (2021) Development of MWCNT decorated with green synthesized AgNps-based electrochemical sensor for highly sensitive detection of BPA. J Appl Electrochem 51(3):447-462. https://doi.org/10.1007/s10800-020-01511-3

63. Papavassiliou GC (1976) Surface plasmons in small Au-Ag alloy particles. J Phys F: Met Phys 6(4):L103. https://doi.org/10.1088/ 0305-4608/6/4/004

64. Li J, Liu J-C, Gao C-J (2010) On the mechanism of conductivity enhancement in PEDOT/PSS film doped with multi-walled carbon nanotubes. J Polym Res 17(5):713-718. https://doi.org/10.1007/ s10965-009-9360-1

65. Alizadeh M, Mehmandoust M, Nodrat O, Salmanpour S, Erk N (2021) A glassy carbon electrode modified based on molybdenum disulfide for determination of folic acid in the real samples. J Food Measur Charact: 1-8. https://doi.org/10.1007/ s11694-021-01128-w

66. Sen S, Sarkar P (2015) A novel third-generation xanthine biosensor with enzyme modified glassy carbon electrode using electrodeposited MWCNT and nanogold polymer composite film.
RSC Adv 5(116):95911-95925. https://doi.org/10.1039/C5RA1 8889J

67. Kaladevi G, Wilson P, Pandian K (2020) Silver nanoparticledecorated PANI/reduced graphene oxide for sensing of hydrazine in water and inhibition studies on microorganism. Ionics 26(6):3123-3133. https://doi.org/10.1007/s11581-020-03457-0

68. Manal A, Azab SM, Hendawy HA (2019) A facile nano-iron oxide sensor for the electrochemical detection of the anti-diabetic drug linagliptin in the presence of glucose and metformin. Bull Natl Res Cent 43(1):1-8. https://doi.org/10.1186/s42269-019-0132-8

69. Mehmandoust M, Çakar S, Özacar M, Salmanpour S, Erk N (2021) Electrochemical sensor for facile and highly selective determination of antineoplastic agent in real samples using glassy carbon electrode modified by 2D-MoS2 NFs/TiO2 NPs. Top Catal: 1-13. https://doi.org/10.1007/s11244-021-01479-0

70. Allahverdiyeva S, Yunusoğlu O, Yardım Y, Şentürk Z (2021) First electrochemical evaluation of favipiravir used as an antiviral option in the treatment of COVID-19: a study of its enhanced voltammetric determination in cationic surfactant media using a boron-doped diamond electrode. Anal Chim Acta 1159:338418. https://doi.org/10.1016/j.aca.2021.338418

71. Mohamed MA, Eldin GM, Ismail SM, Zine N, Elaissari A, Jaffrezic-Renault N, Errachid A (2021) Innovative electrochemical sensor for the precise determination of the new antiviral COVID19 treatment Favipiravir in the presence of coadministered drugs. J Electroanal Chem 895:115422. https://doi.org/10.1016/j.jelec hem.2021.115422

72. Morsy MI, Nouman EG, Abdallah YM, Zainelabdeen MA, Darwish MM, Hassan AY, Gouda AS, Rezk MR, Abdel-Megied AM, Marzouk HM (2021) A novel LC-MS/MS method for determination of the potential antiviral candidate favipiravir for the emergency treatment of SARS-CoV-2 virus in human plasma: application to a bioequivalence study in Egyptian human volunteers. J Pharm Biomed Anal 199:114057. https://doi.org/10.1016/j.jpba. 2021.114057

Publisher's note Springer Nature remains neutral with regard to jurisdictional claims in published maps and institutional affiliations. 